\section{$\underset{\substack{\text { hommes } \\ \text { \& migrations }}}{ }$}

\section{Hommes \& migrations}

Revue française de référence sur les dynamiques

migratoires

$1283 \mid 2010$

Cuisines et dépendances

\title{
Partage et transmission de la cuisine marocaine en France
}

\author{
Marie Poinsot et Fatéma Hal
}

\section{(2) OpenEdition \\ Journals}

\section{Édition électronique}

URL : http://journals.openedition.org/hommesmigrations/983

DOI : 10.4000/hommesmigrations.983

ISSN : 2262-3353

\section{Éditeur}

Musée national de l'histoire de l'immigration

\section{Édition imprimée}

Date de publication : 1 janvier 2010

Pagination : 24-31

ISBN : 978-2-919040-04-9

ISSN : $1142-852 X$

\section{Référence électronique}

Marie Poinsot et Fatéma Hal, «Partage et transmission de la cuisine marocaine en France », Hommes \& migrations [En ligne], 1283 | 2010, mis en ligne le 29 mai 2013, consulté le 01 mai 2019. URL : http:// journals.openedition.org/hommesmigrations/983; DOI : 10.4000/hommesmigrations.983 


\section{Partage et transmission de la cuisine marocaine en France}

Entretien réalisé par Marie Poinsot avec Fatéma Hal, ethnologue de formation, propriétaire du restaurant Le Mansouria dans le Xle arrondissement à Paris et auteure de nombreux ouvrages sur la gastronomie marocaine

Depuis une vingtaine d'années, Fatéma Hal, la créatrice du restaurant

Le Mansouria dans le $\mathrm{Xl}^{\mathrm{e}}$ arrondissement de Paris, s'est plongée dans la cuisine marocaine et ses traditions. L'expérience de la migration a conduit cette ambassadrice érudite et passionnée à apprendre, à transmettre et à partager un savoir culinaire qui, à moins d'être pratiqué, est menacé d'oubli. De la culture à l'assiette, le langage des saveurs et des savoir-faire offre le terrain d'une rencontre permanente. 


\section{Pouvez-vous nous rappeler votre parcours, depuis votre formation en ethnologie, en passant par l'ouverture de votre restaurant et la rédac- tion de vos ouvrages sur la gastronomie?}

Je suis arrivée en France dans les années soixante-dix, où on m'a mariée à un immigré. Ce n'était pas un mariage arrangé, et mon arrivée correspond à celle de n'importe quelle femme immigrée qui rejoint son mari, sauf que le mien était né en France d'une mère française.

Je me suis retrouvée à Paris, en banlieue, comme tout le monde, à habiter dans un HLM. Nous avons reçu un jour un cousin qui m'a dit : "Tu étais tellement brillante, tu ne vas pas simplement passer ta vie à élever tes enfants dans une cité HLM! Tu peux t'inscrire à l'université."

Cette phrase m'a touchée. Je n'avais même pas le bac, j’ai donc travaillé pour avoir l'équivalence et je suis ensuite entrée à l'université Paris 8 , où j'ai commencé à préparer une licence de littérature arabe. C'était une belle époque, on croyait pouvoir changer le monde. Nous ne l'avons pas changé finalement, mais nous nous sommes changés nous-mêmes.

Par la suite, je me suis inscrite à l'École pratique des hautes études. La directrice de mes recherches était Camille Lacoste-Dujardin, la femme du géopoliticien Yves Lacoste. Entre ces deux périodes d'études, j'ai travaillé à l'ICEI et à l'ADRI, où je dirigeais un programme national pour les femmes. Ce programme regroupait des femmes maghrébines et portugaises, en vue de créer des associations en France. J'ai fini par en monter une à Grasse, dans les Alpes-Maritimes, qui a organisé le premier festival de cinéma arabe, avec l'aide de la mairie et de l'assistante sociale de l'époque. C'était vraiment un tout petit festival, nous avons dû faire du porte à porte pour faire venir du monde.

C'est comme cela que je suis "montée dans le train", et j'ai poursuivi mon chemin entre l'université et mon travail, parce que j'avais envie d'apprendre tout en continuant à travailler. J'ai milité dans les banlieues, j'ai donné des cours de langue arabe, j'ai fait de l'animation culturelle, de l'interprétariat, etc. Juste après cela, on m'a proposé un poste intéressant comme conseillère technique au ministère des Droits des femmes. À l'époque, j’avais donc deux emplois à mi-temps : je travaillais comme conseillère au ministère des Femmes et comme rédactrice-informatrice dans des îlots sensibles, pendant deux ans.

Cependant, tous les emplois qu'on me proposait étaient des contrats à durée déterminée et, finalement, après des contrats de un ou deux ans, je me suis retrouvée au chômage. Je me suis alors dit : "Pourquoi ne pas créer ma propre entreprise? Qu'estce que je peux et sais faire?"J'ai pensé à ouvrir un restaurant, parce que, lorsque je faisais des petits dîners avec les moyens du bord, on me disait toujours que c'était très bon. 
En 1984, forte de cette assurance et motivée, j'ai essayé de contacter et de rencontrer des banquiers. Or ils m'envoyaient balader, car je n'avais pas un centime à l'époque. Les premières questions qu'ils me posaient étaient systématiquement : "Quel est votre apport personnel et quelles sont vos garanties ?” Je n'avais rien à répondre.

J'ai alors eu une idée toute simple, celle de vendre des repas à l'avance, un peu comme cela se faisait à l'époque pour les théâtres et les cinémas avec les souscriptions, à la différence près que, contrairement aux cinémas, je n'avais même pas de locaux! De plus, mon contrat au ministère des Femmes se terminait et je me retrouvais avec peu de moyens. Par ailleurs, j'avais récupéré mes enfants qui vivaient auparavant avec leur père. Je devais donc choisir entre payer mon loyer ou nourrir mes enfants. J'étais dans une situation compliquée : je ne payais pas mon loyer et aucune banque ne voulait me prêter de l'argent. Grâce à cette idée de vendre des repas à l'avance, des associations m'ont prêté des lieux pour organiser des dîners. Voilà comment j'ai monté mon restaurant.

\section{À l'époque, y avait-il beaucoup de restaurants marocains à Paris ?}

Non, il y en avait très peu sur Paris - pour ne pas dire aucun -, pour la simple raison qu'on parlait alors de "restaurant oriental" ou on disait : "On va manger dans un couscous."

\section{Ensuite, vous vous êtes spécialisée dans l'étude de la gastronomie comme phénomène culturel ?}

Je me suis rendu compte du rôle important de la mémoire et de la transmission des savoir-faire culinaires. Le fait de dire que les personnes originaires du Maghreb ont une tradition orale et que tout se transmet oralement m'énervait, dans la mesure où des livres importants existaient sur le sujet, mais étaient méconnus en France. Au départ, je voulais juste comprendre pourquoi lorsque l'on parle du Maghreb ou des femmes on affirme souvent des choses fausses. Il est vrai, cependant, que la cuisine se transmet oralement de mère en fille avec, malheureusement, un certain appauvrissement de la gastronomie ces dernieres décennies.

Au départ, mon travail consistait à aller sur le terrain pour enregistrer les vieilles femmes en train de parler de cuisine. Je me suis retrouvée avec un coffre entier de cassettes. J'écoutais leurs recettes, mais j'ai compris tout de suite que la cuisine ne se réduisait pas à cela. Chez nous, lorsqu'on demande comment préparer un plat, on donne les ingrédients et les quantités, sans préciser de mesures. De quoi la femme interviewée avait-elle envie de me parler? Cela m’amusait de faire des travaux sur ces aspects méconnus de notre culture. Les intellectuels français ne s'occupent pas de la cuisine, qui reste une affaire de femmes; comme si les affaires de femmes 
n'étaient pas intéressantes. Voilà ce qui m’a poussée à aller de plus en plus loin et m’a donné envie d'en apprendre plus.

\section{En observant les familles marocaines arrivées en France depuis les années vingt, pensez-vous qu'il existe un véritable souci de préservation des pratiques alimentaires, pour faire de la cuisine le ciment de l'identité marocaine en France ?}

Je pense que la transmission ne se fait pas seulement par la confection des plats, mais aussi par le goût. Les hommes goûtent et parlent aussi. Hommes et femmes, jeunes et vieux, tous ont donc un rôle dans la transmission du goût. Le rôle des immigrés dans la préservation de la culture est majeur. Ce sont peut-être les immigrés qui ont conservé le plus cette identité, beaucoup plus que les intellectuels. Concrètement, il s'agit d'une véritable action de survie, qui ne concerne pas que les immigrés, mais l'humanité tout entière.

Quand on se trouve "en dehors", dans les saveurs et les gestes, la dernière chose qui reste dans la migration est la langue, la langue qui goûte et qui parle. Les migrants voyagent avec une partie d'eux-mêmes et dans les quartiers se créé une forme d'organisation sociale liée à leur installation : on fait le couscous le vendredi parce qu'on a le temps, mais aussi parce que c'est un jour de partage, symbolique. La 
menthe est facile à faire pousser, les navets et les carottes montrent comment on adopte et adapte les produits de l'autre, qui ne sont pas si différents des nôtres. L'immigré voyage avec des graines de blé, parce qu'il croît que l'autre n'en a pas, et c'est le cas pour bien d'autres ingrédients encore.

Un épisode m'a marquée : ma belle-mère m'a préparé du pain pour le porter à ma mère au Maroc lors d'un voyage au pays. Elle faisait du pain "de chez nous", alors que j'étais dans un pays, dans une ville, où tous les matins on prépare du pain ! J'ai compris seulement après que c'était pour elle une manière de lui dire : "Vous voyez, je continue à faire du pain, je ne suis pas perdue."

\section{Dans les bagages des migrants, quels sont les ustensiles emportés ?}

Le plus simple est le kesmetré que les immigrés emportent dans leurs bagages pour le couscous. Par la suite, j'ai découvert que des migrants originaires de Marnia, pas loin de Oujda, étaient à l'origine de la création d'un nouvel ustensile qui a marqué l'immigration maghrébine. Pour faire le pain, on se servait normalement du plat à tajine en terre. Mais en France, avec le gaz de ville, on ne pouvait pas obtenir facilement

Cela raconte une jolie histoire, le couscous

devient le plat partagé et préféré des Français, c'est-à-dire un patrimoine commun. la braise nécessaire pour une cuisson dans ce type de plat. Les maris qui travaillaient dans des fonderies ont donc fabriqué un plat en fonte - comme un poêle - qui remplaçait le tajine en terre. Ce plat a ensuite transité par Oujda. Au Maroc, on l'appelle ainsi "le plat d'Oujda", alors que ce sont des immigrés algériens de la région de Marnia qui ont été les premiers à le fabriquer. J'ai suivi un peu le cheminement de ce plat, pour voir comment, à partir de rien, une société s'organise autour de la nourriture. Si la plupart des voyageurs, des diplomates ou des intellectuels se déplacent avec leur cuisinier ou préfèrent sortir dans un restaurant à Paris, l'immigré, lui, reste avec ses enfants chez lui, avec un sentiment de dépaysement par rapport à la société française. Pour lui, le seul moyen de dire qu'il existe, la seule chose qui lui reste en dehors de l'usine, c'est cette nourriture, cette transmission du goût, cette occasion de parler encore une fois de la mère ou de la grand-mère.

\section{En dehors du couscous, y a-t-il d'autres plats qui servent de "marqueurs identitaires"?}

Le couscous n'est plus vraiment un plat identitaire, il est désormais un plat français. Par exemple, j'ai vu à New York des restaurants proposant le "french couscous". Cela 
raconte une jolie histoire, le couscous devient le plat partagé et préféré des Français, c'est-à-dire un patrimoine commun.

Le tajine représente le coté raffiné du Maroc, alors qu'à la base c'est un plat berbère extraordinaire. Au Maroc, on appelle ainsi le plat qui sert pour la préparation de ces mets, même si désormais la fonte ou d'autres matériaux ont substitué l'ustensile en terre traditionnel. Pour le reste, les Français disent souvent que les pâtisseries "orientales" ne sont pas bonnes, ce qui est vrai pour la plupart des pâtisseries commercialisées, ce qui trahit aussi le désir d'une rencontre culinaire. Or cette rencontre culinaire entre les sociétés française et maghrébine est la seule que les migrants ont vraiment réussie.

\section{J'imagine qu'il y a un rituel alimentaire autour du mois du ramadan et de la rupture du jeûne. Prépare-t-on des plats différents, qui ont une valeur pour ce temps fort de l’année?}

La religion de l'islam est souvent associée en France à la violence des islamistes. Or, pendant le ramadan, j'ai eu envie, il y a deux ans, de publier le livre Ramadan, cuisine du partage. J'ai été étonnée de voir l'intérêt et le formidable accueil réservé à cet ouvrage. Ceci pour dire que le ramadan est aussi un mois d'élégance, de goût, de partage. Il y a le rêve, le voyage, le parfum. Et l'invitation adressée à l'autre. C'est un mois extraordinaire qui donne presque envie aux autres de faire le ramadan. J'ai vu souvent mes copains français ne pas manger, faire le jeûne avec nous, pour profiter de la rupture du jeûne le soir, car ils avaient envie de tout goûter ! On est dans le registre: "C'est ma nourriture, je te la fais connaître, je la partage avec toi, je propose toutes mes spécialités", et non plus dans celui de savoir à qui appartiennent ces recettes.

\section{De mère en fille ou de mère en fils, arrive-t-on encore à partager ces savoirs, à les transmettre, à donner le goût de faire aux jeunes géné- rations?}

Si la transmission a pratiquement disparu en Occident, chez nous elle est bloquée. Le premier obstacle est celui du temps disponible, car les femmes, heureusement de plus en plus scolarisées, ont de moins en moins de temps pour la cuisine. Le deuxième obstacle vient de la faible reconnaissance de la cuisine marocaine dans la société française. Dans les écoles hôtelières, les jeunes ne se forment pas à cette cuisine. Si on ne fait pas quelque chose, il faut craindre à terme une extinction progressive de cette transmission.

Le même phénomène se retrouve au Maroc même, car si la France a réglé la question de la transmission de la gastronomie par l'apprentissage dans les écoles hôtelières, au Maroc rien de cela n'existe. De plus, la cuisine qu'on apprend dans ces 
écoles ne correspond peut-être pas à celle de la mère, elle s'est transformée. Nous avons donc un énorme travail à faire pour préserver cet héritage culinaire. Par ailleurs, la gastronomie marocaine est reconnue à l'étranger, car lorsque la France reconnaît une cuisine, les autres pays généralement suivent, la France étant reconnue dans le monde comme une sorte de patrie du goût. En France, plusieurs personnes "portent le drapeau" de la cuisine marocaine.

\section{Les Marocains en France sont-ils actifs dans la création d'entreprises et de restaurants proposant un cuisine de qualité ?}

Je pense qu'on a eu une grande chance au départ. En 1985, lorsque j'ai ouvert mon restaurant, on parlait de "cuisine orientale". Ensuite, il a suffi que quelqu'un "porte le drapeau" de la cuisine marocaine pour que la presse en parle. En réalité, l'histoire de la cuisine marocaine et alégrienne en France n'est pas une question liée à l'ordre d'arrivée. Contrairement à ce que l'on peut croire, la cuisine algérienne est très riche, de même que ses déclinaisons, comme la cuisine algéroise.

\section{La valorisation d'une cuisine plutôt qu'une autre est donc due à des personnes qui en sont devenues les "porte-drapeau" ?}

Oui, et pour preuve, il n'y a qu'à regarder les magasins La bague de Kenza qui proposent des pâtisseries algériennes dans Paris. Maintenant, on parle de 
“pâtisseries algériennes". La même chose peut se dire de la cuisine française : s'il n'y avait pas les grands restaurants et les grands chefs connus dans le monde entier, la cuisine française ne serait peut-être pas si renommée.

\section{Comment la cuisine intègre-t-elle une entité culturelle? Comment peut-elle donner une autre image des migrants marocains ?}

La cuisine a toujours été intégrée dans une vision globale de la culture, seules les personnes qui n'ont pas de culture ignorent cela. La cuisine fait partie de la culture, des textes, de l'art, de l'écriture, de la chanson, de la peinture, de la vie quotidienne, mais aussi du politique, de l'économique, du social. Des guerres ont été déclenchées pour une poignée de poivre ou pour la domination de la route des épices.

\section{Comment intéresser le public à ce thème, avec quelles initiatives (expositions, conférences, etc.) ?}

Je pense qu'il faudrait travailler sur les textes avec les personnes qui étudient ces questions, emprunter le chemin le plus classique et traditionnel, celui que l'on prend habituellement pour sensibiliser à la musique ou à la littérature. En effet, souvent, on a l'impression que parler de cuisine est exceptionnel, or ce n'est pas vrai. La cuisine est une chose fabuleuse dans la mesure où elle fait partie de la vie beaucoup plus que la musique ou l'écriture. Si on ne lit pas, on ne meurt pas, si on n'écoute pas de musique, on ne meurt pas, tandis que si on ne mange pas, là on meurt !

La cuisine appartient aussi au domaine de l'affectif. Lorsqu'une personne dit : " $M a$ mère fait le meilleur couscous", cela n'est pas toujours vrai, parfois c'est même immangeable, mais en disant cela, on parle de l'amour que l'on porte à sa propre mère, c'est fabuleux.

Après, il s'agit juste d'un travail de valorisation et de présentation. Il faut se donner les mêmes moyens que ceux qui existent pour faire connaître des formes artistiques comme la littérature ou la peinture, car c'est le même type de travail. Souvent, on ne parle pas de cuisine, car on est complexés. Par exemple, si on me présente comme anthropologue, c'est beaucoup plus valorisant que si on me présente comme cuisinière. Or c'est justement cette vision des choses qu'il faut changer. 